\title{
Proliferation of Fibroblast Cells in Periradicular Tissue Following Intentional Replantation of Vertical Root Fractures Using Two Materials
}

\author{
R Tri Endra Untara ${ }^{1}$, Widjijono ${ }^{2}$, Widya Asmara ${ }^{3}$, Diatri N Ratih ${ }^{4}$
}

\begin{abstract}
Aim: This study aimed to investigate the fibroblast cells proliferation in periradicular tissue using mineral trioxide aggregate (MTA) and selfadhesive resin cement as the adhesive material for vertical root fracture fragments following intentional replantation.

Materials and methods: This study used 27 male New Zealand rabbits aged 8-12 weeks. The mandibular incisor of each rabbit was extracted, and to simulate vertical fracture, the incisor tooth was sectioned vertically from the cervical to the $2 / 3$ apical. The samples were randomly divided into three groups of nine each. Group 1 (control group), no application of any material. Group 2, the fracture line was sealed using MTA. Group 3 , with self-adhesive resin cement. All teeth in all groups were then inserted back (replanted) into the socket of the rabbits. Each group was further divided into three subgroups according to the duration of replantation, namely, group A: week 1, group B: week 2, and group C: week 3 . Rabbits were sacrificed according to each duration of replantation for histological preparations. The number of fibroblast cells was evaluated by counting at the three viewpoints under the light-microscope (400× magnification) and Optilab camera; finally, the calculation results were averaged. Data were analyzed using a two-way ANOVA and post hoc LSD test, with a significance level of $95 \%$.

Results: Following MTA application in the third week produced the highest number of fibroblast cells $(104+29.5)$ compared to other groups. Conversely, the lowest number of fibroblast cells occurred in the control group in the first week of observation $(4.33+3.5)$.

Conclusion: MTA produced the greatest fibroblast cell proliferation than self-adhesive resin cement, particularly in week 3 of vertical root fractures replantation.

Clinical significance: As the adhesive material for vertical root fracture fragments, MTA generated greater fibroblast proliferation than selfadhesive resin cement. Therefore, it is recommended to use MTA to attach vertical root fracture fragments.

Keywords: Fibroblasts, Intentional replantation, Mineral trioxide aggregate, Self-adhesive resin cement, Vertical root fractures.

The Journal of Contemporary Dental Practice (2021): 10.5005/jp-journals-10024-3169
\end{abstract}

\section{INTRODUCTION}

Trauma to teeth and their supporting tissues is a challenge in dental practice. One of the impacts of trauma on the teeth is induced vertical fracture, defined as a fracture that occurs vertically on the long axis of the crown or root of a tooth. This trauma can cause the tooth to loosen or come out of its socket (avulsion). ${ }^{1,2}$ Many irritants then will enter and occupy the fracture space and adjacent canals, and this space will also become a hiding place for bacteria and their products. The periodontal tissue will become inflamed, and connective tissue will grow into a fracture to the root canal. ${ }^{3,4}$

In cases of vertical root fracture, the root of the tooth usually remains embedded and attached to its socket. Therefore, it is often required to perform intentional replantation, which is described as extracting intentionally of the tooth, followed by root canal treatment and finally returning the tooth to the same socket in one visit. ${ }^{5,6}$ The joining of fracture fragments is very important to support the success of treatment. The choice of materials and techniques is very crucial that are able to create a density for sealing the gaps between the fragments, leads to no more empty gaps that will be occupied by liquids or bacteria. ${ }^{7}$

Currently, a new type of resin adhesive called self-adhesive resin cement has been introduced to the market. This material does not require pretreatment on the tooth surface to be attached, and the application is completed in one step. The advantage of utilizing this material is simplifying the treatment and reducing the handling time of replanted teeth with vertical

\author{
1,2,4 Faculty of Dentistry, Universitas Gadjah Mada, Yogyakarta, \\ Indonesia \\ ${ }^{3}$ Faculty of Veterinary Medicine, Universitas Gadjah Mada, Yogyakarta, \\ Indonesia
}

Corresponding Author: Diatri N Ratih, Faculty of Dentistry, Universitas Gadjah Mada, Yogyakarta, Indonesia, Phone: +622742882270; +6281802654009, e-mail: diatri@ugm.ac.id

How to cite this article: Untara RTE, Widjijono, Asmara W, et al. Proliferation of Fibroblast Cells in Periradicular Tissue Following Intentional Replantation of Vertical Root Fractures Using Two Materials. J Contemp Dent Pract 2021;22(9):998-1002.

Source of support: Nil

Conflict of interest: None

root fractures. ${ }^{8}$ Although the clinical success is more than $90 \%$ at 2 years posttreatment, ${ }^{9}$ the use of composite resins in intentional replantation treatment is required to be evaluated its success histologically to determine the regeneration of periradicular tissue. It is because of residual toxic monomers and the inherent shrinkage property of composite resin. ${ }^{10,11}$ These phenomena may affect the healing or regeneration of periradicular tissue.

Mineral trioxide aggregate (MTA) has many beneficial properties in treating endodontic cases, such as biocompatibility, closure density, mechanical strength, and the capacity for healing periradicular tissue. ${ }^{12,13}$ It is likely to enhance the success of vertical 
root fracture treatment when using MTA; hence, it is needed to prove the use of MTA as material to seal the vertical root fracture.

One of the factors that determine the outcome of injury healing is the proliferation of fibroblast. ${ }^{14,15}$ However, only a few studies are available in the literature investigating the effect of using MTA and self-adhesive resin cement as the adhesive materials for vertical fracture tooth fragments on the number of fibroblast cells in periradicular tissue. Thus, the present study aimed to investigate the fibroblast cells proliferation in periradicular tissue using MTA and self-adhesive resin cement as the adhesive material for vertical root fracture fragments following intentional replantation.

\section{Materials and Methods}

\section{Sample Selection}

This study was conducted in the Integrated Research Laboratory, Faculty of Dentistry, Universitas Gadjah Mada, and Pathology Laboratory, Department of Pathology, Faculty of Veterinary Medicine, Universitas Gadjah Mada, Yogyakarta, Indonesia. Ethical clearance for research using experimental animals has been approved by the Research Ethics Commission of the Faculty of Dentistry, Universitas Gadjah Mada number 00566/KKEP/FKG-UGM/EC/2020. This study used 27 male white rabbits, New Zealand, aged 8-12 weeks, and bodyweight of 2500-3000 gm. All rabbits were physically healthy with normal oral cavity conditions and no infection.

\section{Sample Preparation for Vertical Root Fracture and Intentional Replantation}

The rabbits were anesthetized intramuscularly on the thigh with $10 \%$ xylazine ketamine $10 \mathrm{mg} / \mathrm{kg}$ body weight. Before surgery, oral prophylaxis was performed using a $10 \%$ povidone-iodine solution (Betadine, PT Mahakam Beta Farma, Jakarta Timur, Indonesia). Before extraction of the mandibular incisor, the socket of the tooth was widened and enlarged. The tooth was separated from the ligament and then was extracted with the pliers. The crown of the mandibular incisor was cut half of the incisal to avoid contact with the antagonist's tooth.

Furthermore, the tooth was sectioned longitudinally parallel to the tooth axis in a mesial-distal direction from coronal to apical using a small, high-speed fissure bur under a saline spray (PT Otsuka Indonesia, Yogyakarta, Indonesia). The vertical fracture was induced by splitting up to two-third of the root length from the cervical region and leaving one-third of the apical tooth intact. ${ }^{16}$ The pulp tissue was extirpated using barbed broach (Dentsply Sirona, Charlotte, North Carolina, United States of America); the tooth was then irrigated using sterile saline $(0.9 \% \mathrm{NaCl}, \mathrm{PT}$. Otsuka, Semarang, Indonesia).

Afterward, the rabbits were then divided into three groups of nine each. Group 1 served as the control group, with no application of any material. Group 2, the fracture line was sealed using MTA (White ProRoot MTA, Dentsply Sirona, United States of America). Group 3 was the same as Group 2, but the fracture line was sealed with self-adhesive resin cement (Rely X Unicem, 3M ESPE, St Paul, Minnesota, United States of America). All teeth of the rabbits in all groups were then inserted back (replanted) into the socket of the rabbits. Flowable resin composite (Filtek Z350 XT Flowable, 3M ESPE, United States of America) was used to fixate the replanted teeth from detachment from their socket. All rabbits were injected using penicillin at $40-80,000 \mu / \mathrm{kg}$ IM. Rabbits were then sacrificed according to each duration of replantation, namely, group A: week 1, group B: week 2, and group C: week 3.

\section{Sample Preparation for Histological Observation}

Rabbits were euthanized using an injection of ketamine xylazine at a dose of $10 \mathrm{mg} / \mathrm{kg}$ body weight, and their jaw in the incisor region was dissected according to the observation time, namely week 1 , week 2, and week 3 . Hematoxylin \& Eosin (H\&E) staining was performed using an Automatic Staining Machine (Thermoscientific, Waltham, Massachusetts, United States of America). The sequence of HE staining was as followed: the deparaffinization process was carried out by inserting the preparations into xylol three times for 5 minutes each. The specimens then were gradually rehydrated with 100, 95, 80, and $70 \%$ alcohol for 2 minutes, respectively. The remaining alcohol was removed by placing the specimen in distilled water. The specimens were immersed in hematoxylin solution for 7 minutes, resulting in the appearance of the blue color of the cell nucleus. Eosin staining was performed using eosin solution for 0.5-1 minute, then washed with distilled water to remove the remaining eosin solution, providing a red color as a contrast to the cytoplasm. Counterstain was dehydrated with 70, 80, 95, and $100 \%$ alcohol for 4 minutes, respectively. The specimens were immersed in xylol 1, 2, and 3 solutions each for 2 minutes to provide tissue transparency.

The number of fibroblast cells was assessed by three examiners previously calibrated, and the Kappa test was employed to determine the agreement between the examiners (Kappa $\geq 0.75$ ). The fibroblast was observed by counting the number of fibroblast cells per field of view using a light microscope (Nikon YS 100, Japan) equipped with an Optilab advances camera (Optilab, PT. Miconos, Yogyakarta, Indonesia) with a magnification of $400 \times$. The calculation was done by examining the three viewpoints in each treatment group, and then the calculation results were averaged.

\section{Statistical Analysis}

Data obtained were subjected to statistical analysis using one-way ANOVA, followed by a post hoc LSD test, and were processed and analyzed using the SPSS Version 21 program. The level of significance was $95 \%$.

\section{Results}

Table 1 shows the mean and standard deviation of the number of fibroblasts observed at week 1 , week 2 , and week 3 postintentional replantation of vertical root fractures with the application of MTA and adhesive resin cement. The mean scores for the control group at weeks 1, 2, and 3 were $4.33+3.5,24.67+10.5,52.33+12.1$. The mean scores for MTA at weeks 1, 2, and 3 were $39.67+16.6$, $40.33+8.4,104+29.5$. The average values for self-adhesive resin cement at weeks 1, 2, and 3 were $74.67+30.9,39+15.9,41+5.6$.

Based on the week of observation, the control group had an increasing number of fibroblasts. Similar to the control group, the

Table 1:The mean and standard deviation of the number of fibroblasts observed at week 1 , week 2 , and week 3 postintentional replantation of vertical root fractures with the application of MTA and self-adhesive resin cement

\begin{tabular}{lccccc}
\hline No. & Group & $n$ & Week 1 & Week 2 & Week 3 \\
\hline 1 & Group 1 (control) & 3 & $4.33 \pm 3.5$ & $24.67 \pm 10.5$ & $52.33 \pm 12.1$ \\
2 & Group 2 & 3 & $39.67 \pm 16.6$ & $40.33 \pm 8.4$ & $104 \pm 29.5$ \\
3 & Group 3 & 3 & $74.67 \pm 30.9$ & $39 \pm 15.9$ & $41 \pm 5.6$ \\
\hline
\end{tabular}


MTA group also produced an increased number of fibroblasts from week 1 to week 2 . On the other hand, in the self-adhesive resin cement group, the number of fibroblasts decreased from week 1 to week 3 (Fig. 1).

Figures 2 to 4 are histological observations from representative specimens that show the number of fibroblasts. It can be seen that the number of fibroblasts in the control group was the lowest at week 1 , whereas the number of fibroblasts using MTA as an adhesive to seal vertical root fractures was the highest at week 3 . The highest number of fibroblasts occurred in self-adhesive resin cement at week 1.

Two-way ANOVA statistical analysis (Table 2) showed that there was a significant effect of observation time (weeks) and the materials used, as well as the interaction between the time of observation and the materials used on the number of fibroblasts $(p<0.05)$. The inference of the present study indicates that MTA at week 3 produced the highest number of fibroblast cells compared to the self-adhesive resin cement and control group.

\section{Discussion}

In this study, fibroblasts were used as an indicator since they play an important role in the injury healing process or regeneration. Tooth extraction on intentional replantation may cause damage to the periodontal ligament, which led to injury to the tooth socket. ${ }^{17}$ According to Maddaluno et al., ${ }^{14}$ fibroblasts play a crucial factor in tissue repair, from the final inflammatory phase to complete epithelization of injured tissue, by secreting growth factors, cytokines,

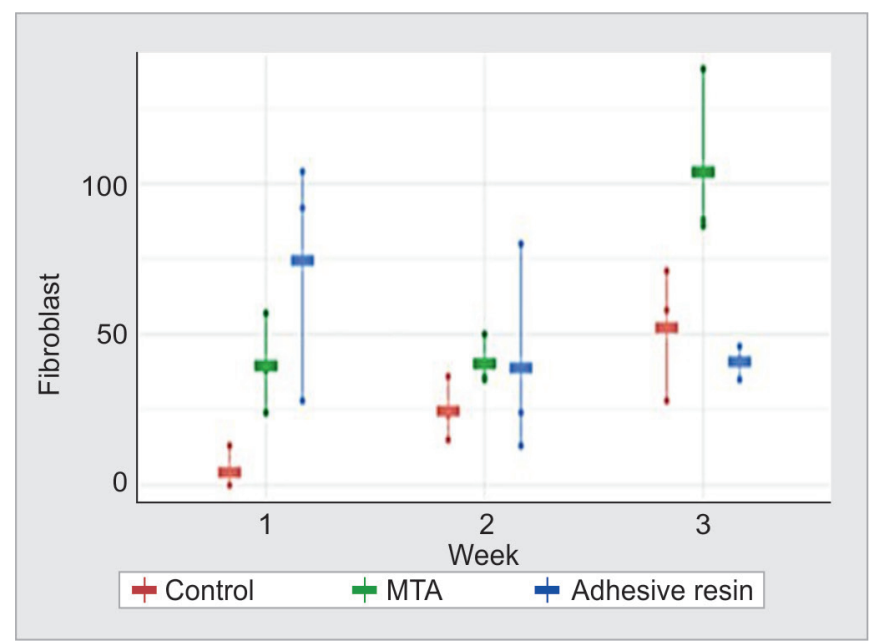

Fig. 1: The graph of the mean number of fibroblasts on observation week 1 , week 2 , and week 3 using MTA

Table 2: The results of the two-way ANOVA of the number of fibroblasts observed at week 1 , week 2 , and week 3 postintentional replantation of vertical root fractures with the application of MTA and self-adhesive resin cement

\begin{tabular}{lccccc}
\hline Treatment & $d f$ & $\begin{array}{c}\text { Sum of } \\
\text { squares }\end{array}$ & $\begin{array}{c}\text { Average of } \\
\text { squares }\end{array}$ & $F$ & $p$ \\
\hline Time & 2 & 5038.222 & 2519.111 & 4.665 & $0.018^{*}$ \\
Material used & 2 & 5592.889 & 2796.444 & 5.179 & $0.014^{*}$ \\
Interaction & 4 & 9046.889 & 2261.722 & 4.189 & $0.014^{*}$ \\
\hline
\end{tabular}

${ }^{*}$ Statically significant $(p<0.05)$ collagen, and extracellular matrix components. At the same time, fibroblast migration and proliferation perform a vital function in the healing process by initiating a proliferative phase of repair.

The control group showed that observation time affected the increased number of fibroblasts from week 1 to week 3 , although the mean number was lower than the other two treatment groups. This phenomenon indicated healing or regeneration process of the soft tissue around the replanted tooth occurred continuously. A tooth with its roots has a similar composition as the hard tissue in the body; hence there is no refusal reaction of the body against the tooth re-inserting into its socket. ${ }^{18}$ This present study showed that the lower mean number of fibroblasts occurred in the control group compared to other treatment groups throughout the observation. This phenomenon is possible because the fractured root was not sealed by any materials in the control group. Consequently, the surgery area and its surrounding tissues became less sterile due to the drinking and eating activities of the rabbits that may affect the healing process while they were treated until sacrificed. The most important factor in ensuring the healing of an injury is the problem of preventing bacterial contamination and infection. The injury can be healed not only due to the rabbit's endurance but also due to the aid of antibacterial injection. Prevention of injury infection is a requirement for the occurrence of the normal proliferation of tissue cells to cover the injury. ${ }^{19}$

Group 2, which was sealed with MTA, exhibited that observation time caused the rise in the number of fibroblasts from week 1 to week 3, and the mean number of fibroblasts on MTA application was the highest compared to the other two groups; even the mean number of fibroblasts at week $3(104+29.5)$ was more than double compared to week $2(40.33+8.4)$. This result indicates that MTA has a significant effect on the proliferation of fibroblast during the observation time. The number of fibroblasts increased throughout observation time, revealing that MTA is biocompatible. These results are in accordance with previous studies, which stated that MTA has high biocompatibility to human cells. ${ }^{12,13}$ Another investigator also showed that the 12-week of observation time in animal tissue either in vitro or in vivo study exhibited normal cell growth and no infection. ${ }^{20}$

Group 3, which was sealed with self-adhesive resin cement, demonstrated that observation time influenced the number of fibroblasts. It is shown that a decrease in the number of fibroblasts decrease from week 1 to week 2 and then slightly increased at week 3 ; however, the number was lower than the control and MTA group. This condition indicates that the self-adhesive resin cement during observation time induced an unfavorable effect of the periradicular tissue regeneration process on the vertical root fractures. The unfavorable effect of self-adhesive resin cement on periodontal tissue might be due to the presence of cytotoxic components, which would affect the regeneration of injured tissue. The cytotoxic components, which are contained in self-adhesive resin cement, are TEGMA and fluoride. ${ }^{21}$

In contrast to the present study, Liying et al. ${ }^{9}$ performed intentional replantation treatment of vertical fractures of human posterior teeth using separate bonding agents and composite resins, and the teeth were examined clinically and radiographically 2 years after replantation. The results showed that the sealing of vertically fractured teeth using a separate dentin bonding system and composite resin was effective. Another study by Unver et al. ${ }^{6}$ also reported the use of adhesive resin, namely, 4-methacryloxyethyl trimellitate anhydride/methacrylate-tri- $n$-butyl borane (4-META/MMA-TBB) 

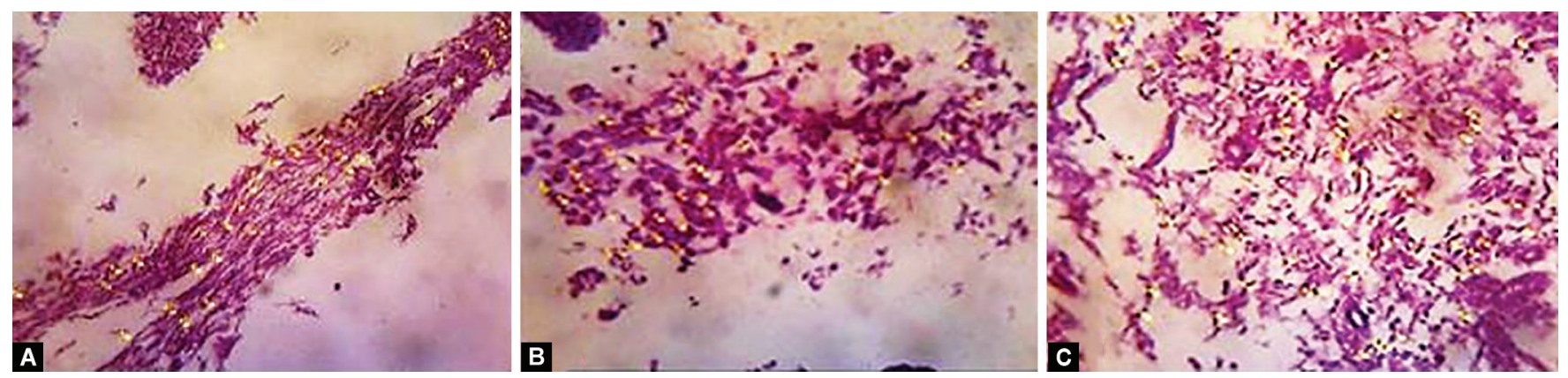

Figs $2 A$ to C: Diagram of the mean number of fibroblasts on observation (A) Week 1; (B) Week 2; and (C) Week 3 using MTA
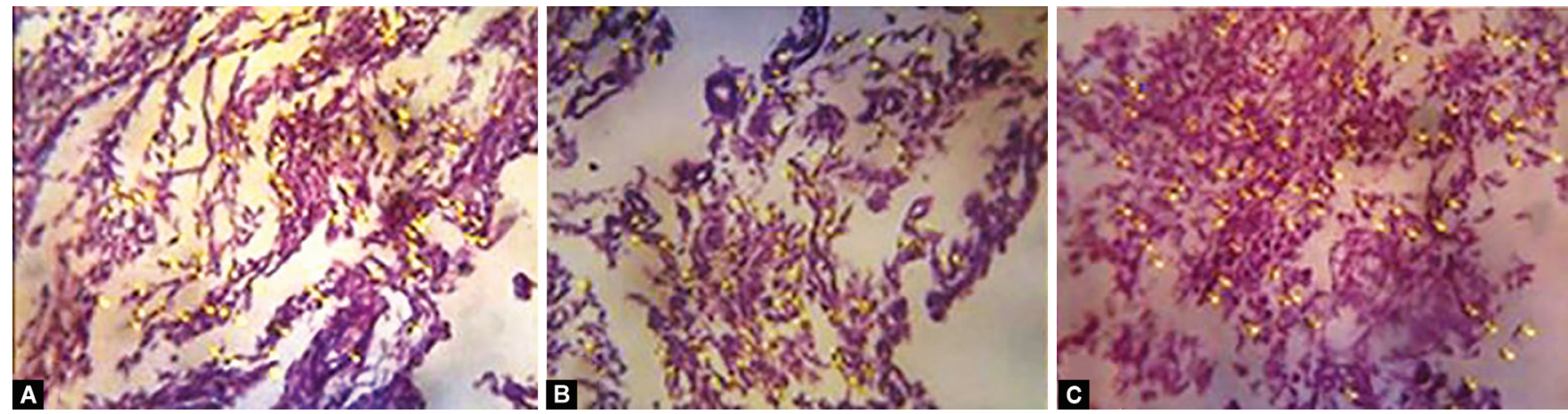

Figs $3 A$ to C: Histological images exhibiting the number of fibroblasts of the control group (no application of material at vertical root fracture) (A) At week 1; (B) Week 2; and (C) Week 3 (H\&E stain at 400× magnification)
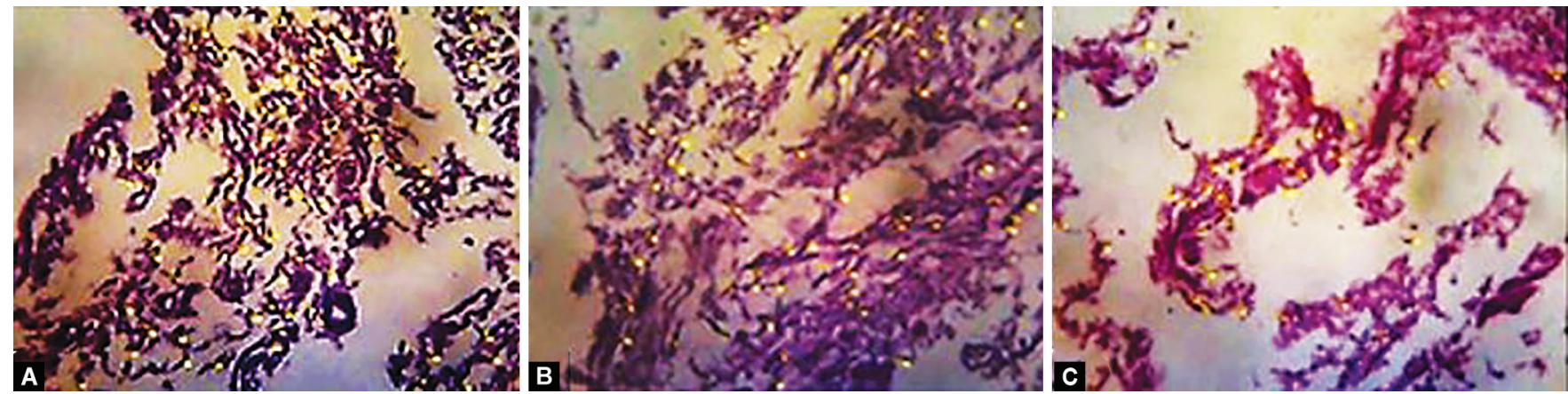

Figs 4A to C: Histological images demonstrating the number of fibroblasts using MTA as an adhesive material to seal vertical root fracture (A) At week 1; (B) Week 2; and (C) Week 3 (H\&E stain at 400× magnification)

resin cement, to attach the vertically fracture fragments of the human tooth in intentional replantation. The results showed the clinical success of the treatment. However, both treatments performed by Liying et al..$^{9}$ and Unver et al. ${ }^{6}$ did not examine the teeth' periradicular tissues histologically to determine the occurrence of tissue regeneration.

In this present study, self-adhesive resin cement was inserted to seal the gaps along the fracture line and filling the entire root canal space. Although the activation of the LED light has been carried out to harden the cement, there remain parts of self-adhesive resin cement that were not directly exposed to the LED light. Consequently, the material was not polymerized completely during exposure to LED light. If resin cement was not polymerized completely, residual monomer components, such as Bis-GMA, UDMA, TEGDMA, camphorquinone, and HEMA might be leaching out to the surrounding area. ${ }^{22,23}$ These residual monomers may potentially cause tissue injury and inhibit tissue healing. Additionally, these monomers are cytotoxic to pulp and gingival cells. ${ }^{11}$

The limitations of the present study are that the findings are the use of experimental rabbits for each treatment group and observation time using different rabbits. It is because each treatment required cutting of the rabbit's jaw for histological observation; hence each rabbit was required to be sacrificed. Even though age and weight control were carried out; however, each rabbit had an immune system that was different among them. Thus, the defense reaction of each rabbit to heal the wound and to respond to the material for sealing the gap along the fracture line may be 
different. To resolve the problem, this study used only one type of experimental animal (rabbit) and comparing with a control group. Other clinical trials are necessary to be undertaken to explain the efficacy of materials used on the proliferation of fibroblast.

\section{Conclusion}

This study concluded that MTA produced the greatest fibroblast cell proliferation than self-adhesive resin cement, particularly in week 3 of vertical root fractures in intentional replantation. Thus, it is recommended to use MTA rather than self-adhesive resin cement for attaching vertical root fracture fragments.

\section{REFERENCES}

1. Patnana AK, Kanchan T. Tooth fracture. Treasure Island, FL: Stat Pearls Publishing; 2020; p. 1-8.

2. Benoist FL, Seck A, Benoist HM. 42-month follow-up of double root fracture of a lateral mandibular incisor. Eur Endod J 2017;26(2):2-5. DOI: 10.5152/eej.2017.17021.

3. Khasnis SA, Kidiyoor KH, Patil AB, et al. Vertical root fractures and their management. J Conserv Dent 2014;17(2):103-110. DOI: 10.4103/09720707.128034

4. Dhawan A, Gupta S, Mittal R. Vertical root fractures: an update review. J Res Dent 2014;2(3):107-113. DOI: 10.4103/2321-4619.143457.

5. Kumar ARP, Shemesh $H$, Jothilatha $S$, et al. Diagnosis of vertical root fractures in restored endodontically treated teeth: A timedependent retrospective cohort study. J Endod 2016;42(8):1175-1180. DOI: 10.1016/j.joen.2016.04.012.

6. Unver S, Onay EO, Ungor M. Intentional re-plantation of a vertically fractured tooth repaired with an adhesive resin, Int Endod J 2011;44:1069-1078. DOI: 10.1111/j.1365-2591.2011.01922.x.

7. Becker B. Intentional replantation techniques: a critical review. J Endod 2018;44(1):14-21. DOI: 10.1016/j.joen.2017.08.002.

8. Maño EP, Algarra RM, Fawzy A, et al. In vitro bonding performance of modern self-adhesive resin cements and conventional resinmodified glass ionomer cements to prosthetic substrates. Appl Sci 2020;10(8157):1-15. DOI: 10.3390/app10228157.

9. Liying $Y$, Beiyun $X$, Bin $W$, et al. Clinical research on treatment of vertically fractured posterior teeth by intentional replantation using dentin bonding and composite resin. J Osaka Dent Univ 2004;38(2):101-107. DOI: 10.18905/jodu.38.2_101.

10. Kamalak H, Taghizadehghalehjoughi A, Kamalak A. The biocompatibility of resin composite materials on different stem cells. Biomed Res 2019;30(1):32-41. DOI: 10.35841/ biomedicalresearch.30-18-1162.

11. Gajewski VES, Pfeifer CS, Froes-Salgado NR, et al. Monomers used in resin composites: degree of conversion, mechanical properties and water sorption/solubility. Braz Dent J 2012;23(5):508-514. DOI: 10.1590/S0103-64402012000500007.

12. Parirokh $\mathbf{M}$, Torabinejad $\mathbf{M}$. Mineral trioxide aggregate: a comprehensive literature review-part III: clinical applications, drawbacks, and mechanism of Action. J Endod 2010;36(3):400-413. DOI:10.1016/j.joen.2009.09.009.

13. Nagmode PS, Satpute AB, Patel AV, et al. The effect of mineral trioxide aggregate on the periapical tissues after unintentional extrusion beyond the apical foramen. Case Rep Dent 2016;2016:1-5. DOI: 10.1155/2016/3590680.

14. Maddaluno L, Urwyler C, Werner S. Fibroblast growth factors: key players in regeneration and tissue repair. Development 2017;144(22):4047-4060. DOI: 10.1242/dev.152587.

15. Addis R, Cruciani S, Santaniello S, et al. Fibroblast proliferation and migration in wound healing by phytochemicals: Evidence for a novel synergic outcome. Int J Med Sci 2020;17(8):1030-1042. DOI: 10.7150/ ijms.43986.

16. Szabo Z, Bradley K, Cahalane AK. Rabbit soft tissue surgery. Vet Clin Exot Anim 2016;19(1):159-188. DOI: 10.1016/j.cvex.2015.08.007.

17. Marouane $O$, Turki $A$, Oualha $L$, et al. Tooth replantation: an update. Med Buccale Chir Buccale 2017;23(2):103-110. DOI: 10.1051/ $\mathrm{mbcb} / 2016056$.

18. Nemcovsky CE, Weinreb M. Soft and hard tissue regeneration. Dent J 2018;6(4):1-4. DOI: 10.3390/dj6010004.

19. Yellanki SK, Singh J, Manfi FV. Formulation, characterization and evaluation of metronidazole gel for local treatment of periodontitis. Int J Pharm Bio Sci 2010;1(2):1-8. DOI: 10.22376/ijpbs.2010.1.2.1.

20. Upadhyay A, Pillai S, Khayambashi $P$, et al. Biomimetic aspects of oral and dentofacial regeneration. Biomimetics 2020;125(4):51. DOI: 10.3390/biomimetics5040051.

21. Şişmanoğlu S, Demirci M, Schweikl H, et al. Cytotoxic effects of different self-adhesive resin cements: cell viability and induction of apoptosis. J Adv Prosthodont 2020;12:89-99. DOI: 10.4047/ jap.2020.12.2.89.

22. Klein-Júnior CA, Zimmer R, Hentschke GS, et al. Effect of heat treatment on cytotoxicity of self-adhesive resin cements: cell viability analysis. Eur J Dent 2018;12(2):281-286. DOI: 10.4103/ejd. ejd_34_18.

23. Sajnani AR, Hegde MN. Leaching of monomers from bulk-fill composites: an in vitro study. J Conserv Dent 2016;19(13):482-486. DOI: 10.4103/0972-0707.190020. 